ISSN 1855-3966 (printed edn.), ISSN 1855-3974 (electronic edn.)

ARS MATHEMATICA CONTEMPORANEA 21 (2021) \#P2.03

https://doi.org/10.26493/1855-3974.2260.c0e

(Also available at http://amc-journal.eu)

\title{
A generalization of balanced tableaux and marriage problems with unique solutions
}

\author{
Brian Tianyao Chan * \\ KeyFi, First Floor, First St. Vincent Bank Ltd. Building James Street, Kingstown, \\ St. Vincent and the Grenadines
}

Received 22 February 2020, accepted 24 February 2021, published online 30 September 2021

\begin{abstract}
We consider families of finite sets that we call flagged and that have been characterized by Chang as being the families of sets that admit unique solutions to Hall's marriage problem and we consider generalizations of Edelman and Greene's balanced tableaux previously investigated by Viard. In this paper, we introduce a natural generalization of Edelman and Greene's balanced tableaux that involves families of sets that satisfy Hall's marriage condition and certain words in $[\mathrm{m}]^{n}$, then prove that flagged families can be characterized by a strong existence condition relating to this generalization. As a consequence of this characterization, we show that the arithmetic mean of the sizes of subclasses of such generalized tableaux is given by a generalization of the hook-length formula.
\end{abstract}

Keywords: Balanced tableaux, Hall's marriage condition, shelling.

Math. Subj. Class. (2020): 05A20, 05C70, 05E45

\section{Introduction}

Hall's Marriage Theorem is a combinatorial theorem proved by Hall [11] that asserts that a finite family of sets has a transversal if and only if this family satisfies the marriage condition. This theorem is known to be equivalent to at least six other theorems which include Dilworth's Theorem, Menger's Theorem, and the Max-Flow Min-Cut Theorem [20]. Hall Jr. proved [10] that Hall's Marriage Theorem also holds for arbitrary families of finite sets, where by arbitrary we mean families of finite sets that do not necessarily have a finite number of members. Afterwards, Chang [3] noted how Hall Jr.'s work in [10] can be used to characterize marriage problems with unique solutions.

*The author would like to thank Stephanie van Willigenburg for her guidance and advice during the development of this paper. Furthermore, the author gratefully acknowledges the insightful advice and feedback from the anonymous referees. The author was supported in part by the Natural Sciences and Engineering Research Council of Canada [funding reference number PGSD2 - 519022 - 2018]. https://sites.google.com/view/btchan

E-mail address: bchan600@gmail.com (Brian Tianyao Chan) 
Standard skew tableaux are well-known and intensively studied in algebraic combinatorics, for example [15, 18, 19, 21]. Moreover, another class of tableaux was introduced by Edelman and Greene in [5, 4], where they defined balanced tableaux on partition shapes. In investigating the number of maximal chains in the weak Bruhat order of the symmetric group, Edelman and Greene proved [5, 4] that the number of balanced tableaux of a given partition shape equals the number of standard Young tableaux of that shape. Since then, connections to random sorting networks [1], the Lascoux-Schützenberger tree [16], and a generalization of balanced tableaux pertaining to Schubert polynomials [7] have been explored.

In this paper we consider a new perspective for marriage problems with unique solutions by interpreting such objects as shapes for generalized tableaux. Specifically we call the families of finite sets that admit marriage problems with unique solutions flagged and give a new characterization of these families of sets in Theorem 3.10. In this characterization, we generalize standard skew tableaux and Edelman and Greene's balanced tableaux to families with systems of distinct representatives, we generalize hook sets to members of such families, and we generalize bijective fillings of tableaux to certain words in $[m]^{n}$. We then use our characterization of marriage problems with unique solutions to show in Theorem 3.25 that the arithmetic mean of the sizes of subclasses of such generalized tableaux is given by a generalization of the hook-length formula. The hook-length formula was discovered by Frame, Robinson, and Thrall and they proved that it enumerates the number of standard Young tableaux of a given partition shape [8]. The formula consists of parameters known as hook-lengths. Subsequent to Frame, Robinson and Thrall's work, hook-lengths have been shown to be connected to many known properties of tableaux. They are integral, for instance, in work by Edelman and Greene on balanced tableaux [4] and in results established by Morales, Pak, and Panova [17, 18]. Properties of Edelman and Greene's balanced tableaux and related notions are of interest [6, 7]. Moreover, generalizations of balanced tableaux were investigated by Viard. In [24, 23], Viard proved what is equivalent to the following which we state using the terminology in this paper. If $\mathcal{F}$ is a flagged family, if $t$ is a transversal of $\mathcal{F}$, and if $f$ is a configuration of $t$, then there exists a permutation $\sigma$ that satisfies $f$. Moreover, Viard proved [24] what is equivalent to the following which we also state using the terminology in this paper. Let $S$ be a finite subset of $\mathbb{N}^{2}$ and let $\mathcal{F}$ be the family of hooks $\left\{H_{(i, j)}:(i, j) \in S\right\}$ where $H_{(i, j)}=\{(i, j)\} \cup\left\{\left(i, j^{\prime}\right) \in S: j^{\prime}>j\right\} \cup\left\{\left(i^{\prime}, j\right) \in S: i^{\prime}>i\right\}$. Furthermore, let $t$ be the transversal of $\mathcal{F}$ defined by $t\left(H_{(i, j)}\right)=(i, j)$ for all $(i, j) \in S$. Then the average value of $A_{n, n}(f)$ over all configurations $f$ of $t$ satisfying $A_{n, n}(f) \geq 1$ is given by the hooklength formula $n ! / \prod_{(i, j) \in S} h_{(i, j)}$ where $h_{(i, j)}=\left|H_{(i, j)}\right|$ for all $(i, j) \in S$. Afterwards, we indicate how our generalization of standard skew tableaux and balanced tableaux can be analysed using Naruse's Formula for skew tableaux and how such an approach can be extended to skew shifted shapes $[9,17,19]$ and likely to certain $d$-complete posets $[9,19]$.

\section{Preliminaries}

Throughout this paper, let $\mathbb{N}$ denote the set of positive integers and for all $n \in \mathbb{N}$, define $[n]=\{1,2, \ldots, n\}$. For all $X^{\prime} \subseteq X$, let the restriction of $f$ to $X^{\prime}$, which we denote by $\left.f\right|_{X^{\prime}}$, be the function $g: X^{\prime} \rightarrow Y$ defined by $g(r)=f(r)$ for all $r \in X^{\prime}$. For all $m, n \in \mathbb{N}$, say that a function $f:[n] \rightarrow[m]$ is order-preserving if for all $1 \leq i \leq j \leq n, f(i) \leq$ $f(j)$. Lastly, we write examples of permutations using one-line notation. When describing 
families of sets, call $F \in \mathcal{F}$ a member of $\mathcal{F}$. We treat families of sets as multisets, so the members of $\mathcal{F}$ are counted with multiplicity. That is, $|\mathcal{F}|=|I|$ if $\mathcal{F}=\left\{F_{i}: i \in I\right\}$.

An illustrative class of examples that we use in this paper will come from skew shapes. Hence, we recall them below and describe the notation we will use. A partition $\lambda$ is a weakly decreasing sequence of positive integers. We write $\lambda=\left(\lambda_{1}, \lambda_{2}, \ldots, \lambda_{\ell}\right)$ to denote such a partition, where $\lambda_{i} \in \mathbb{N}$ for all $1 \leq i \leq \ell$. If $\lambda$ is a partition, then we will also represent it as a Young diagram, which we also denote by $\lambda$. Specifically, the Young diagram of $\lambda=\left(\lambda_{1}, \lambda_{2}, \ldots, \lambda_{\ell}\right)$ is a subset of $\mathbb{N}^{2}$ defined by

$$
\bigcup_{i=1}^{\ell}\left\{(i, j): 1 \leq j \leq \lambda_{i}\right\} .
$$

Moreover, if $\lambda$ and $\mu$ are Young diagrams such that $\mu \subset \lambda$, then define a skew shape $\lambda / \mu$ to be the set $\lambda \backslash \mu$. We also consider a Young diagram $\lambda$ as the skew shape $\lambda / \mu$ where $\mu$ is the empty partition. We use the English convention for depicting Young diagrams and skew shapes. In order to follow this convention, we call the elements of $\lambda / \mu$ the cells of $\lambda / \mu$, the non-empty subsets of the form $\left\{\left(i^{\prime}, j^{\prime}\right) \in \lambda / \mu: i^{\prime}=i\right\}$ the rows of $\lambda / \mu$, and the non-empty subsets of the form $\left\{\left(i^{\prime}, j^{\prime}\right) \in \lambda / \mu: j^{\prime}=j\right\}$ the columns of $\lambda / \mu$.

\section{Flagged families of sets and words in $[\mathrm{m}]^{n}$}

We investigate families of sets that satisfy Hall's marriage condition and generalizations of Edelman and Greene's balanced tableaux by proving relationships between these classes of structures. In Section 3.1, we introduce marriage problems with unique solutions as flagged families and generalizations of balanced tableaux, then we give a new characterization of marriage problems with unique solutions in terms of these tableaux. In Section 3.2, we explain how our results relate to tableaux on skew shapes. Lastly, in Section 3.3, we show that the arithmetic mean of the sizes of subclasses of the above generalized tableaux is given by a generalization of the hook-length formula.

\subsection{A new characterization}

A well-known notion for families of sets is the following.

Definition 3.1 (Folklore [14]). Let $n \in \mathbb{N}$, and let $\mathcal{F}$ be a finite family of subsets of $[n]$. Then a transversal of $\mathcal{F}$ is an injective function $t: \mathcal{F} \rightarrow[n]$ such that $t(F) \in F$ for all $F \in \mathcal{F}$. The set $\{t(F): F \in \mathcal{F}\}$ is called a system of distinct representatives of $\mathcal{F}$.

Families of sets that have transversals are of great interest. Exemplary of this is Hall's Marriage Theorem, which we present below.

Definition 3.2 (Marriage condition, Hall [11]). Let $n \in \mathbb{N}$, and let $\mathcal{F}$ be a finite family of subsets of $[n]$. Then $\mathcal{F}$ satisfies the marriage condition if for all subfamilies $\mathcal{F}^{\prime}$ of $\mathcal{F}$,

$$
\left|\mathcal{F}^{\prime}\right| \leq\left|\bigcup_{F \in \mathcal{F}^{\prime}} F\right| .
$$

Theorem 3.3 (Marriage Theorem, Hall [11]). Let $n \in \mathbb{N}$, and let $\mathcal{F}$ be a family of nonempty subsets of $[n]$. Then $\mathcal{F}$ has a transversal if and only if $\mathcal{F}$ satisfies the marriage condition. 
In order to meaningfully use the families of sets in Hall's Marriage Theorem, we will define more structure on them.

Definition 3.4. Let $n \in \mathbb{N}$, let $\mathcal{F}$ be a family of non-empty subsets of $[n]$, and let $t$ be a transversal of $\mathcal{F}$. Then a configuration $f$ of $t$ is a function $f:[n] \rightarrow \mathbb{N}$ such that for all $F \in \mathcal{F}$,

$$
f(t(F)) \leq|F| .
$$

Moreover, for $m \in[n]$, a surjective map $\sigma:[n] \rightarrow[m]$ satisfies $f$ if for all $F \in \mathcal{F}$, the positive integer $\sigma(t(F))$ is the $k^{t h}$ smallest element of the set $\sigma(F)$, where $k=f(t(F))$.

Example 3.5. Let $\mathcal{F}=\left\{F_{1}, F_{2}\right\}$ be a family of sets on [2] where $F_{1}=[2]$ and $F_{2}=[2]$. The injective function $t: \mathcal{F} \rightarrow[2]$ defined by $t\left(F_{1}\right)=1$ and $t\left(F_{2}\right)=2$ is a transversal of $\mathcal{F}$. Consider three configurations $f^{\prime}, f^{\prime \prime}$, and $f^{\prime \prime \prime}$ of $t$ defined by $f^{\prime}(1)=1$ and $f^{\prime}(2)=1$, $f^{\prime \prime}(1)=1$ and $f^{\prime \prime}(2)=2$, and $f^{\prime \prime \prime}(1)=2$ and $f^{\prime \prime \prime}(2)=2$.

Note $\sigma:[2] \rightarrow[1]$ satisfies $f^{\prime}$ because $\sigma(1)=1$ is the smallest element of $\sigma\left(F_{1}\right)=$ $\sigma([2])=[1]$ and because $\sigma(2)=1$ is the smallest element of $\sigma\left(F_{2}\right)=\sigma([2])=[1]$. However, no permutation $\sigma:[2] \rightarrow[2]$ can satisfy $f^{\prime}$. It can also be checked that the surjective map $\sigma:[2] \rightarrow[1]$ and the permutation $\sigma=21$ do not satisfy $f^{\prime \prime}$ but the permutation $\sigma=12$ satisfies $f^{\prime \prime}$. Moreover, for all $m \in[2]$ and for all surjective maps $\sigma:[2] \rightarrow[m], \sigma$ does not satisfy $f^{\prime \prime \prime}$.

Now, we define the following stronger form of the marriage condition.

Definition 3.6 (cf. [3]). Let $n \in \mathbb{N}$, let $\mathcal{F}$ be a finite family of subsets of $[n]$, and write $m=|\mathcal{F}|$. Then $\mathcal{F}$ is flagged if there exists a bijection $\sigma_{\mathcal{F}}:[m] \rightarrow \mathcal{F}$ such that for all $k \in[m]$,

$$
\left|\bigcup_{i=1}^{k} \sigma_{\mathcal{F}}(i)\right|=k .
$$

Informally, $\sigma_{\mathcal{F}}$ maps each $k$ to a subset, such that the union of the first $k$ subsets has cardinality $k$.

In [3], Chang noted the following as a simple consequence of Hall Jr.'s work ([10], Theorem 2).

Proposition 3.7 (Chang [3]). If $n \in \mathbb{N}$, then a finite family $\mathcal{F}$ of subsets of $[n]$ has exactly one transversal if and only if $\mathcal{F}$ is flagged.

In particular, by Theorem 3.3, all flagged families satisfy the marriage condition. The families of sets $\mathcal{F}$ in Proposition 3.7 are referred to as marriage problems with unique solutions $[13,12]$.

Remark 3.8. When describing a flagged family $\mathcal{F}$, we will use total orderings on the members of this family by fixing orderings $F_{1}, F_{2}, \ldots, F_{n}$ of the members of $\mathcal{F}$ that satisfy

$$
\mathcal{F}=\left\{F_{i}: 1 \leq i \leq n\right\},
$$

and, for all $1 \leq k \leq n$,

$$
\left|\bigcup_{i=1}^{k} F_{i}\right|=k .
$$


We observe that no flagged family is a multi-set. Let $\mathcal{F}$ be a flagged family and fix an ordering $F_{1}, F_{2}, \cdots, F_{n}$ of the members of $\mathcal{F}$ as described in Remark 3.8. Suppose that $F_{j}=F_{j^{\prime}}$ for some $j<j^{\prime}$. Then,

$$
\bigcup_{i=1}^{j} F_{i}=\bigcup_{i=1}^{j^{\prime}} F_{i}
$$

contradicting Equation (3.1) of Definition 3.6.

Before proving the main result of this paper, we prove the following lemma.

Lemma 3.9. Let $\mathcal{F}$ be a flagged family of subsets of $[n]$. Moreover, let $S \subseteq[n]$ be the set of elements $k \in[n]$ such that $k \in F$ for exactly one member $F$ of $\mathcal{F}$. Then $S$ is not empty.

Proof. Let $m=|\mathcal{F}|$. Because $\mathcal{F}$ is flagged, Definition 3.6 and Equation (3.1) imply that there exists a bijection $\sigma_{\mathcal{F}}:[m] \rightarrow \mathcal{F}$ and an element $k \in[n]$ such that

$$
\bigcup_{i=1}^{m-1} \sigma_{\mathcal{F}}(i)=\left(\bigcup_{i=1}^{m} \sigma_{\mathcal{F}}(i)\right) \backslash\{k\} .
$$

So as $k \in \sigma_{\mathcal{F}}(m)$ and as, for all $1 \leq i<m, k \notin \sigma_{\mathcal{F}}(i)$, it follows that $k \in S$ and that $S$ is non-empty.

Now, we prove the main result of this paper.

Theorem 3.10. Let $n \in \mathbb{N}$, let $\mathcal{F}$ be a family of subsets of $[n]$ such that $|\mathcal{F}|=n$, assume that $\mathcal{F}$ satisfies the marriage condition, and let $t$ be a transversal of $\mathcal{F}$. Moreover, let $S \subseteq[n]$ be the set of elements $k \in[n]$ such that $k \in F$ for exactly one member $F$ of $\mathcal{F}$. Lastly, let $m$ be an integer satisfying

$$
\min (n, n-|S|+1) \leq m \leq n .
$$

Then $\mathcal{F}$ is flagged if and only if for all configurations $f$ of $t$, there exists a surjective map $\sigma:[n] \rightarrow[m]$ such that $\sigma$ satisfies $f$.

Proof. Let $n, \mathcal{F}, t, S$, and $m$ be as described in the theorem. First assume that for all configurations $f$ of $t$, there exists a surjective map $\sigma:[n] \rightarrow[m]$ that satisfies $f$. If $n=1$, then the only family of $\{1\}$ with a transversal is the family $\mathcal{F}=\{\{1\}\}$, which is flagged.

So assume without loss of generality that $n \geq 2$. Consider the configuration $f_{1}$ of $t$ defined by $f_{1}(t(F))=|F|$ for all $F \in \mathcal{F}$. By assumption, there exists a surjective map $\sigma^{\prime}:[n] \rightarrow[m]$ that satisfies $f_{1}$. Moreover, let $k \in[n-1]$, and assume that we can fix an ordering $\mathcal{F}=\left\{F_{i}^{\prime}: i \in[n]\right\}$ of $\mathcal{F}$ so that the following holds for all integers $0 \leq j \leq k-1$.

$$
\left|\bigcup_{i=1}^{n-j} F_{i}^{\prime}\right|=n-j
$$

Note that Equation (3.2) holds if $k=1$ because the fact that $\mathcal{F}$ has a transversal implies that $\bigcup_{F \in \mathcal{F}} F=[n]$.

Next, let $1 \leq s \leq n-k+1$ satisfy

$$
\sigma^{\prime}\left(t\left(F_{s}^{\prime}\right)\right)=\max _{1 \leq j \leq n-k+1} \sigma^{\prime}\left(t\left(F_{j}^{\prime}\right)\right) .
$$


Suppose that there exists an element $j \in[n]$ such that $1 \leq j \leq n-k+1, j \neq s$, and $t\left(F_{s}^{\prime}\right) \in F_{j}^{\prime}$. By Equation (3.3), $\sigma^{\prime}\left(t\left(F_{j}^{\prime}\right)\right) \leq \sigma^{\prime}\left(t\left(F_{s}^{\prime}\right)\right)$. So as $t\left(F_{s}^{\prime}\right) \in F_{j}^{\prime}$ and $t\left(F_{s}^{\prime}\right) \neq t\left(F_{j}^{\prime}\right)$, it follows that for some $1 \leq \ell \leq\left|F_{j}^{\prime}\right|-1, \sigma^{\prime}\left(t\left(F_{j}^{\prime}\right)\right)$ is an $\ell^{\text {th }}$ smallest element of $\sigma^{\prime}\left(F_{j}^{\prime}\right)$. But then, as $f_{1}\left(t\left(F_{j}^{\prime}\right)\right)=\left|F_{j}^{\prime}\right|, \sigma^{\prime}$ does not satisfy $f_{1}$, contradicting the assumption that $\sigma^{\prime}$ satisfies $f_{1}$.

Hence, $t\left(F_{s}^{\prime}\right) \notin F_{i}^{\prime}$ for all $1 \leq i \leq n-k+1$ satisfying $i \neq s$. In particular, fix an ordering $\mathcal{F}=\left\{F_{i}^{\prime \prime}: i \in[n]\right\}$ of the members of $\mathcal{F}$ so that $F_{i}^{\prime \prime}=F_{i}^{\prime}$ if $i>n-k+1$ and $F_{n-k+1}^{\prime \prime}=F_{s}^{\prime}$, where $s$ is as described in the above paragraph. By Equation (3.2) and the fact that $t\left(F_{s}^{\prime}\right) \notin F_{i}^{\prime}$ for all $1 \leq i \leq n-k+1$ satisfying $i \neq s$, it follows that this ordering of the members of $\mathcal{F}$ satisfies the following equation for all integers $0 \leq j \leq k$.

$$
\left|\bigcup_{i=1}^{n-j} F_{i}^{\prime \prime}\right|=n-j
$$

As the choice of $k \in[n-1]$ is arbitrary, it follows that there exists an ordering $\mathcal{F}=$ $\left\{F_{1}, F_{2}, \ldots, F_{n}\right\}$ of $\mathcal{F}$ such that

$$
\left|\bigcup_{i=1}^{k} F_{i}\right|=k
$$

for all $1 \leq k \leq n$. Hence, $\mathcal{F}$ satisfies Equation (3.1) of Definition 3.6. So, by Definition 3.6, $\mathcal{F}$ is flagged.

Next, assume that $\mathcal{F}$ is flagged. We proceed by strong induction on $n$. Because $\mathcal{F}$ is flagged, we will use the total orderings as described in Remark 3.8 to describe the members of this family.

If $n=1$, then the only family of subsets of $\{1\}$ with a transversal is the family $\mathcal{F}=$ $\{\{1\}\}$. Moreover, with $t$ being the transversal of $\mathcal{F}$ defined by mapping $\{1\}$ to 1 , the only configuration $f$ of $t$ is the function $f:\{1\} \rightarrow \mathbb{N}$ defined by $f(1)=1, S=\{1\}$, $\min (n, n-|S|+1)=1$, and the surjective map $\sigma:\{1\} \rightarrow\{1\}$ satisfies $f$.

So assume that $n \geq 2$ and let $f$ be a configuration of $t$. Since $S$ is not empty by Lemma 3.9, $\min (n, n-|S|+1)=n-|S|+1$, implying that $n-|S|+1 \leq m \leq n$. Assume without loss of generality that

$$
S=\left\{n-m^{\prime}+1, n-m^{\prime}+2, \ldots, n\right\}
$$

for some $m^{\prime} \in[n]$. If $m=1$, then $n-|S|+1 \leq 1$, implying that $n=|S|$. Hence, as $|\mathcal{F}|=n$ and $S=[n]$, every element of $[n]$ is contained in exactly one element of $\mathcal{F}$, that is $\mathcal{F}=\{\{k\}: k \in[n]\}$. So in this case, $t(\{k\})=k$ for all $k \in[n]$, the only configuration $f$ of $t$ is the map defined by $f(k)=1$ for all $k \in[n]$, and the surjective map $\sigma:[n] \rightarrow[m]$, defined by $\sigma(k)=1$ for all $k \in[n]$, satisfies $f$. So assume without loss of generality that $m \geq 2$.

Since $n-|S|+1 \leq m \leq n, m$ satisfies the inequality $n-m^{\prime}+1 \leq m \leq n$. As $\mathcal{F}$ is flagged, there is an ordering $F_{1}^{\prime}, F_{2}^{\prime}, \ldots, F_{n}^{\prime}$ of the members of $\mathcal{F}$ such that

$$
\left|\bigcup_{i=1}^{k} F_{i}^{\prime}\right|=k
$$

for all $1 \leq k \leq n$. Define the following subfamilies of $\mathcal{F}$,

$$
\mathcal{F}_{0}=\{F \in \mathcal{F}: t(F) \leq m-1\}
$$


and

$$
\mathcal{F}_{1}=\{F \in \mathcal{F}: t(F) \geq m\} .
$$

We first prove that $\mathcal{F}_{0}$ is flagged. Because $S$ is the set of elements $k \in[n]$ such that $k \in F$ for exactly one member $F$ of $\mathcal{F}$, Equation (3.4) and the fact that $n-m^{\prime}+1 \leq m \leq n$ implies that for all $m \leq k \leq n, k$ is contained in exactly one member of $\mathcal{F}$ and that for all $F \in \mathcal{F}_{1}$,

$$
|F \cap\{m, m+1, \ldots, n\}|=1 .
$$

In particular, $\mathcal{F}_{0}$ is an $(m-1)$-member family of subsets of $[m-1]$. Write

Assume that there exists an integer $1 \leq j \leq n-1$ such that $F_{j}^{\prime} \in \mathcal{F}_{1}$ and $F_{j+1}^{\prime} \in \mathcal{F}_{0}$.

$$
X_{j}=\bigcup_{i=1}^{j-1} F_{i}^{\prime},
$$

where we assume that $X_{j}=\emptyset$ if $j=1$. Since $F_{j}^{\prime} \in \mathcal{F}_{1}, t\left(F_{j}^{\prime}\right) \in\{m, m+1, \ldots, n\}$ and no member of $\mathcal{F}$ other than $F_{j}^{\prime}$ contains $t\left(F_{j}^{\prime}\right)$. Moreover, by Equation (3.5), $\left|F_{j}^{\prime} \cup X_{j}\right|=$ $\left|X_{j}\right|+1$. So as $t\left(F_{j}^{\prime}\right) \in F_{j}^{\prime}$, it follows that $[m-1] \cap\left(F_{j}^{\prime} \cup X_{j}\right)=[m-1] \cap X_{j}$. Since $F_{j+1}^{\prime} \in \mathcal{F}_{0}, F_{j+1}^{\prime} \subseteq[m-1]$. Moreover, by Equation (3.5), $\left|X_{j} \cup F_{j}^{\prime} \cup F_{j+1}^{\prime}\right|=\left|X_{j} \cup F_{j}^{\prime}\right|+1$. It follows that $F_{j+1}^{\prime} \backslash X_{j}=F_{j+1}^{\prime} \backslash\left(X_{j} \cup F_{j}^{\prime}\right)=\{k\}$ for some $k \in[m-1]$, implying that

$$
\left|F_{j+1}^{\prime} \cup X_{j}\right|=\left|X_{j}\right|+1 \text {. }
$$

So the ordering $\mathcal{F}=\left\{F_{1}^{\prime \prime}, F_{2}^{\prime \prime}, \ldots, F_{n}^{\prime \prime}\right\}$ of the members of $\mathcal{F}$, such that $F_{j}^{\prime \prime}=F_{j+1}^{\prime}$, $F_{j+1}^{\prime \prime}=F_{j}^{\prime}$, and $F_{i}^{\prime \prime}=F_{i}^{\prime}$ for all $i \in[n] \backslash\{j, j+1\}$, satisfies the following by Equation (3.5) and Equation (3.6). For all $1 \leq k \leq n$,

$$
\left|\bigcup_{i=1}^{k} F_{i}^{\prime \prime}\right|=k .
$$

Furthermore, $F_{j}^{\prime \prime} \in \mathcal{F}_{0}$ and $F_{j+1}^{\prime \prime} \in \mathcal{F}_{1}$. If there exists an integer $1 \leq j^{\prime} \leq n-1$ such that $F_{j^{\prime}}^{\prime \prime} \in \mathcal{F}_{1}$ and $F_{j^{\prime}+1}^{\prime \prime} \in \mathcal{F}_{0}$, then argue again as above. Repeating this argument at most a finite number of times, we obtain an ordering $\mathcal{F}=\left\{F_{1}, F_{2}, \ldots, F_{n}\right\}$ of the members of $\mathcal{F}$ where

$$
\left|\bigcup_{i=1}^{k} F_{i}\right|=k
$$

for all $1 \leq k \leq n, \mathcal{F}_{0}=\left\{F_{k}: 1 \leq k \leq m-1\right\}$, and $\mathcal{F}_{1}=\left\{F_{k}: m \leq k \leq n\right\}$. In particular, Equation (3.8) holds for all $1 \leq k \leq m-1$, implying that $\mathcal{F}_{0}$ satisfies Equation (3.1) of Definition 3.6. It follows, by Definition 3.6, that $\mathcal{F}_{0}$ is a flagged family of subsets of $[m-1]$.

So consider the ordering $F_{1}, F_{2}, \ldots, F_{n}$ of the members of $\mathcal{F}$ as above and assume without loss of generality that for all $m \leq i \leq n, t\left(F_{i}\right)=i$. Let $t^{\prime}$ be the transversal of $\mathcal{F}_{0}$ defined by $t^{\prime}(F)=t(F)$ for all $F \in \mathcal{F}_{0}$. Moreover, let $f^{\prime}=\left.f\right|_{[m-1]}$, where $\left.f\right|_{[m-1]}$ denotes the restriction of $f$ to $[m-1]$. In particular, $f^{\prime}$ is a configuration of $t^{\prime}$.

Since it is assumed in the theorem that $\min (n, n-|S|+1) \leq m \leq n$, and since a surjective map $\sigma:[n] \rightarrow[m]$ is a permutation if $m=n$, the following holds. Because $\mathcal{F}_{0}$ is flagged, because $\left|\mathcal{F}_{0}\right|=m-1$, and because $|[m-1]|<n$, the induction hypothesis implies that there exists a permutation $\sigma^{\prime}:[m-1] \rightarrow[m-1]$ such that $\sigma^{\prime}$ satisfies $f^{\prime}$. 
If there exists an integer $m \leq j \leq n$ such that $f(j)=\left|F_{j}\right|$, then there exists a surjective map $\kappa^{\prime}:[n] \rightarrow[m]$ such that $\kappa^{\prime}(i)=i$ for all $1 \leq i \leq m-1$ and the following two properties hold for all $m \leq i \leq n$.

- If $f(i)=\left|F_{i}\right|$, then $\kappa^{\prime}(i)=m$.

- If $f(i)<\left|F_{i}\right|$, then $\kappa^{\prime}(i)$ is equal to the $f(i)^{t h}$ smallest element of $\sigma^{\prime}\left(F_{i} \backslash\{i\}\right)$.

Otherwise, if $f(i)<\left|F_{i}\right|$ for all $m \leq i \leq n$, the following holds. Write $\sigma^{\prime}\left(F_{n} \backslash\{n\}\right)=$ $\left\{r_{1}, r_{2}, \cdots, r_{t}\right\}$ where $t=\left|F_{n}\right|-1$ and $r_{1}<r_{2}<\cdots<r_{t}$. Since $f(n)<\left|F_{n}\right|$, there exists a map $\kappa^{*}:[m-1] \rightarrow[m]$ such that $\kappa^{*}$ is injective and order-preserving and such that, with $x \in[m] \backslash \kappa^{*}([m-1]), x<\kappa^{*}\left(r_{1}\right)$ if $f(n)=1$ and $\kappa^{*}\left(r_{f(n)-1}\right)<x<\kappa^{*}\left(r_{f(n)}\right)$ if $f(n) \geq 2$. So there exists a surjective map $\kappa^{\prime \prime}:[n] \rightarrow[m]$ such that $\left.\kappa^{\prime \prime}\right|_{[m-1]}=\kappa^{*}$ and such that the following two properties hold.

- If $m \leq i<n$, then $\kappa^{\prime \prime}(i)$ is equal to the $f(i)^{t h}$ smallest element of $\kappa^{\prime \prime}\left(\sigma^{\prime}\left(F_{i} \backslash\{i\}\right)\right)$.

- If $i=n$, then $\kappa^{\prime \prime}(i) \notin \kappa^{\prime \prime}([m-1])$ and $\kappa^{\prime \prime}(i)$ is equal to the $f(i)^{t h}$ smallest element of $\kappa^{\prime \prime}(i) \cup \kappa^{\prime \prime}\left(\sigma^{\prime}\left(F_{i} \backslash\{i\}\right)\right)$.

We note that $\left.\kappa^{\prime \prime}\right|_{[m-1]}$ is injective and order-preserving because $\kappa^{*}$ is injective and order-preserving.

So define a surjective map $\kappa:[n] \rightarrow[m]$ as follows. If there exists an integer $m \leq j \leq$ $n$ such that $f(j)=\left|F_{j}\right|$, then define $\kappa=\kappa^{\prime}$. Otherwise, if $f(i)<\left|F_{i}\right|$ for all $m \leq i \leq n$, define $\kappa=\kappa^{\prime \prime}$. Now, define the map $\sigma:[n] \rightarrow[m]$ by

$$
\sigma(i)= \begin{cases}\kappa\left(\sigma^{\prime}(i)\right) & \text { if } 1 \leq i \leq m-1 \\ \kappa(i) & \text { if } m \leq i \leq n\end{cases}
$$

Because $\sigma^{\prime}:[m-1] \rightarrow[m-1]$ is a bijection, the definition of $\kappa$ implies that $\sigma$ is surjective. Moreover, because $\sigma^{\prime}$ satisfies $f^{\prime}$ and because, for all integers $m \leq i \leq n, i$ is contained in exactly one member of $\mathcal{F}$ and $F_{i} \cap\{m, m+1, \ldots, n\}=\{i\}$, the definition of $\kappa$ and the definition of $\sigma$ imply that $\sigma$ satisfies $f$. From this, the theorem follows.

A natural consequence of the above is the following which, combined with Theorem 3.10, gives another characterization of flagged families of sets.

Corollary 3.11. Let $\mathcal{F}$ be a family of subsets of $[n]$ such that $|\mathcal{F}|=n$, assume that $\mathcal{F}$ satisfies the marriage condition, and let $t$ be a transversal of $\mathcal{F}$. Moreover, let $S$ be as in Theorem 3.10. Lastly, let $f_{0}$ be the configuration of $t$ defined by $f_{0}(t(F))=1$ for all $F \in \mathcal{F}$. Then $f_{0}$ is satisfied by some permutation $\sigma:[n] \rightarrow[n]$ if and only if for all integers $n-|S|+1 \leq m \leq n$ and for all configurations $f$ of $t$, there exists a surjective map $\sigma:[n] \rightarrow[m]$ that satisfies $f$.

Proof. Let $f_{1}$ be the configuration of $t$ defined by $f_{1}(t(F))=|F|$ for all $F \in \mathcal{F}$. Then a permutation $\sigma:[n] \rightarrow[n]$ satisfies $f_{0}$ if and only if the permutation $\sigma^{\prime}:[n] \rightarrow[n]$ defined by $\sigma^{\prime}(i)=n-\sigma(i)+1$ for all $i \in[n]$ satisfies $f_{1}$. In particular, $f_{0}$ is satisfied by some permutation if and only if $f_{1}$ is. The first half of the proof of Theorem 3.10 implies that if $f_{1}$ is satisfied by some permutation $\sigma:[n] \rightarrow[n]$, then $\mathcal{F}$ is flagged. Furthermore, by Theorem 3.10, if $\mathcal{F}$ is flagged, then for all integers $n-|S|+1 \leq m \leq n$ and for all configurations $f$ of $t$, there exists a surjective map $\sigma:[n] \rightarrow[m]$ that satisfies $f$. From this, the corollary follows. 
Remark 3.12. A family $\mathcal{F}$ of subsets of $[n]$ such that $\left|\bigcup_{F \in \mathcal{F}} F\right|=|\mathcal{F}|=n$ is called a critical block in [10] by Hall Jr.. He used this notion as a very important ingredient in extending Hall's Marriage Theorem to infinite families of finite sets.

\subsection{The case of skew shapes}

To explain how the results in the previous subsection relate to skew shapes, we will need the following definitions.

Definition 3.13. Let $\lambda / \mu$ be a skew shape with $n$ cells, and let $1 \leq m \leq n$ be an integer. Then a surjective tableau of shape $\lambda / \mu$ is a surjective function $T: \lambda / \mu \rightarrow[m]$ and elements in the range of $T$ are called the entries in $T$. In the case $m=n$ a surjective tableau is a bijective tableau. Moreover, a standard skew tableau of shape $\lambda / \mu$ is a bijective tableau of shape $\lambda / \mu$ such that the entries along every row increase from left to right and the entries along every column increase from top to bottom.

Example 3.14. The tableaux

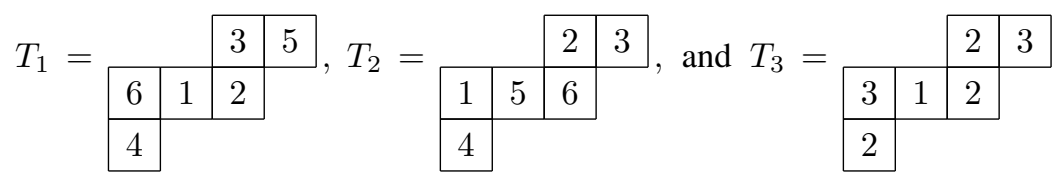

have shape $(4,3,1) /(2)$. Here, $T_{1}$ and $T_{2}$ are bijective and $T_{2}$ is standard. All three are surjective. Moreover, for $T_{1}$ and $T_{2}, m=6$ and for $T_{3}, m=3$.

In order to fully relate Definition 3.13 to Definition 3.4, we will use the following standard definitions.

Definition 3.15. Let $\lambda / \mu$ be a skew shape. For any cell $(i, j) \in \lambda / \mu$, define the corresponding hook $H_{(i, j)}$ to be

$$
H_{(i, j)}=\{(i, j)\} \cup A_{(i, j)} \cup L_{(i, j)},
$$

where

$$
A_{(i, j)}=\left\{\left(i, j^{\prime}\right) \in \lambda / \mu: j^{\prime}>j\right\}
$$

is the $\operatorname{arm}$ of $H_{(i, j)}$ and where

$$
L_{(i, j)}=\left\{\left(i^{\prime}, j\right) \in \lambda / \mu: i^{\prime}>i\right\}
$$

is the leg of $H_{(i, j)}$. Define the corresponding hook-length $h_{(i, j)}$ to be

$$
h_{(i, j)}=\left|H_{(i, j)}\right| .
$$

Example 3.16. Consider the following skew shape $\lambda / \mu$, where $\lambda=(5,4,3,3)$ and $\mu=$ $(2,2,1)$. Then $r=(2,3)$ is the cell of $\lambda / \mu$ depicted below that is filled with a bullet. The entries of $H_{r}$ are filled with asterisks, bullets or circles, so $h_{r}=4$. Moreover, the entry of $A_{r}$ is filled with an asterisk and the entries of $L_{r}$ are filled with circles.

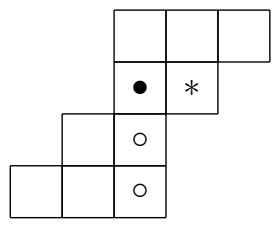


Definition 3.17. Let $\lambda / \mu$ be a skew shape. Then define $\mathcal{F}_{\lambda / \mu}$ to be the set

$$
\left\{H_{r}: r \in \lambda / \mu\right\} \text {. }
$$

Example 3.18. If $\lambda / \mu$ is the skew shape depicted below

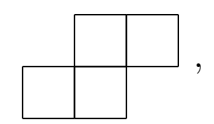

then, as $\lambda / \mu=\{(1,2),(1,3),(2,2),(2,3)\}$,

$$
\mathcal{F}_{\lambda / \mu}=\{\{(1,2),(2,2),(1,3)\},\{(1,3)\},\{(2,1),(2,2)\},\{(2,2)\}\} .
$$

Many families of sets that satisfy the marriage condition are not flagged. For instance, the family $\mathcal{F}=\left\{F_{1}, F_{2}\right\}$, where $F_{1}=F_{2}=\{1,2\}$, satisfies the marriage condition but is not flagged. However, Definition 3.6 is a very broad definition. Let $\lambda$ be a Young diagram. Then an inner corner of $\lambda$ is a cell $r \in \lambda$ such that deleting $r$ from $\lambda$ results in another Young diagram. For instance, if $\lambda=(4,2,2)$, then the inner corners of $\lambda$ are the cells filled with bullets.

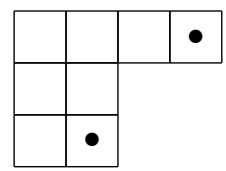

With this definition in mind, let $\lambda / \mu$ be a skew shape with $n$ cells, and consider the family $\mathcal{F}$ of sets defined by $\mathcal{F}=\mathcal{F}_{\lambda / \mu}$. Let $r_{1}, r_{2}, \ldots, r_{n}$ be a sequence of cells in $\lambda / \mu$ such that:

- The cell $r_{1}$ is an inner corner of $\lambda$.

- For all $k \in[n-1]$, the cell $r_{k+1}$ is an inner corner of $\lambda \backslash\left\{r_{1}, r_{2}, \cdots, r_{k}\right\}$.

Define $\sigma_{\mathcal{F}}:[n] \rightarrow \mathcal{F}$ by letting $\sigma_{\mathcal{F}}(k)=H_{r_{k}}$ for all $k \in[n]$. It can be checked that the bijection $\sigma_{\mathcal{F}}$ satisfies Equation (3.1). Now, because the skew shape $\lambda / \mu$ is a finite set, regard $\lambda / \mu$ as being the set $[n]$, where $n$ is the number of cells in $\lambda / \mu$. In particular, regard $\mathcal{F}_{\lambda / \mu}$ as a family of subsets of $[n]$. Then, by the above and by Definition 3.6, $\mathcal{F}$ is flagged. In particular, by Proposition $3.7, \mathcal{F}$ has a unique transversal. The unique transversal $t_{\lambda / \mu}: \mathcal{F} \rightarrow \lambda / \mu$ of $\mathcal{F}$ is given by $t_{\lambda / \mu}\left(H_{r}\right)=r$ for all $r \in \lambda / \mu$.

As we are regarding the cells of a skew shape with $n$ cells as being the elements of $[n]$, we can regard any surjective tableau $T$ of shape $\lambda / \mu$ as being a surjective function $T:[n] \rightarrow[m]$ in which $T(i)=j$ if $j$ is the entry of $T$ in the cell of $T$ corresponding to $i$. Taking $m=n$, we can also regard any bijective tableau of shape $\lambda / \mu$ as being a permutation $T:[n] \rightarrow[n]$. Lastly, as we are regarding the skew shape $\lambda / \mu$ as being the set $[n]$ and as we are regarding $\mathcal{F}_{\lambda / \mu}$ as a family of subsets of $[n]$, we define configurations $f: \lambda / \mu \rightarrow \mathbb{N}$ of $t_{\lambda / \mu}$, where $t_{\lambda / \mu}$ is the unique transversal of $\mathcal{F}_{\lambda / \mu}$, and surjective maps $\sigma: \lambda / \mu \rightarrow[m]$ that satisfy $f$ analogously to Definition 3.4.

Next, let $\lambda / \mu$ be a skew shape with $n$ cells, consider the flagged family of sets $\mathcal{F}_{\lambda / \mu}$, and let $t_{\lambda / \mu}$ be the unique transversal of $\mathcal{F}$. We define the configuration $f_{0}$ of $t_{\lambda / \mu}$ by $f_{0}(r)=1$ for all $r \in \lambda / \mu$. It can be seen that the standard skew tableaux of shape $\lambda / \mu$ are the bijective tableaux of shape $\lambda / \mu$ that satisfy $f_{0}$. Since we regard $\lambda / \mu$ as being the set $[n]$, we can regard $f_{0}$ as being the function $f_{0}:[n] \rightarrow \mathbb{N}$ defined by $f_{0}(k)=1$ for 
all $k \in[n]$. So as we regard $\mathcal{F}_{\lambda / \mu}$ as being a family of subsets of $[n]$, the standard skew tableaux of shape $\lambda / \mu$ can be regarded as being the permutations $\sigma:[n] \rightarrow[n]$ that satisfy $f_{0}$.

Example 3.19. Consider the following surjective tableau of shape $\lambda=(4,3,2)$.

\begin{tabular}{|l|l|l|l|}
\hline 1 & 2 & 3 & 3 \\
\hline 1 & 2 & 3 & \multicolumn{1}{|}{} \\
\cline { 1 - 2 } 3 & 3 & \multicolumn{2}{|c}{} \\
\cline { 1 - 2 } & &
\end{tabular}

Next, consider $\mathcal{F}_{\lambda}$. Let $t_{\lambda}$ be the unique transversal of $\mathcal{F}_{\lambda}$, and let $f: \lambda \rightarrow \mathbb{N}$ be the configuration of $t_{\lambda}$ defined by $f(r)=1$ for all $r \in \lambda / \mu$. It can be checked that the above tableau satisfies $f$.

Edelman and Greene introduced the following class of bijective tableaux, which we re-formulate in terms of the configurations we defined in this paper.

Definition 3.20 (Edelman and Greene [5]). Let $\lambda$ be a Young diagram containing $n$ cells. Moreover, let $t_{\lambda}$ be the transversal of $\mathcal{F}_{\lambda}$ defined by $t_{\lambda}\left(H_{r}\right)=r$ for all $r \in \lambda$ and let $f$ be the configuration defined by

$$
f(r)=\left|L_{r}\right|+1
$$

for all $r \in \lambda$. Then a balanced tableau of shape $\lambda$ is a bijective tableau of shape $\lambda$ that satisfies $f$.

Example 3.21. Let $\lambda=(4,3,2)$, and let $t_{\lambda}$ and $f$ be defined from $\mathcal{F}_{\lambda}$ as described in Definition 3.20. Then

$$
T=\begin{array}{|l|l|l|l|}
\hline 4 & 5 & 8 & 3 \\
\cline { 1 - 2 } 6 & 7 & 9 & \multicolumn{1}{|c}{} \\
\cline { 1 - 2 } 1 & 2 & \multicolumn{2}{|c}{} \\
\cline { 1 - 2 } & &
\end{array}
$$

is balanced because $T$ satisfies $f$. For instance, $f((2,1))=2$ since $L_{(2,1)}=\{(3,1)\}$ and $\left|L_{r}\right|+1=2$. So as $T((2,1))=6, H_{(2,1)}=\{(2,1),(2,2),(2,3),(3,1)\}$, and the set of entries in $T$ that are contained in a cell of $H_{(2,1)}$ is $\{1,6,7,9\}$, it follows that $T((2,1))$ is the $f((2,1))^{t h}$-smallest element of $\{1,6,7,9\}$.

Remark 3.22. The surjective tableaux from Definition 3.13 that satisfy the configuration $f:[n] \rightarrow \mathbb{N}$ defined by $f(k)=1$ for all $k \in[n]$ do not correspond to semistandard tableaux, nor do they correspond to the semistandard balanced labelings in [7].

Balanced tableaux can be regarded as permutations $\sigma:[n] \rightarrow[n]$ that satisfy $f(r)=$ $\left|L_{r}\right|+1$. The function $f(r)=\left|L_{r}\right|+1$ was called the hook rank of $r$ by Edelman and Greene and they used it to define balanced tableaux [5].

Lastly, we give examples illustrating Theorem 3.10 and Corollary 3.11.

Example 3.23. We give an example in which the lower bound $\min (n, n-|S|+1)$ from Theorem 3.10 cannot be improved on. Consider $\lambda=(3,2,1)$. Next, let $\mathcal{F}=\mathcal{F}_{\lambda}$ and let $t$ be the unique transversal of $\mathcal{F}$. As discussed earlier, $\mathcal{F}$ is flagged. Now, let $f$ be the configuration of $\mathcal{F}_{\lambda}$ defined by $f((1,1))=5, f((1,2))=3, f((1,3))=1, f((2,1))=3$, $f((2,2))=1$, and $f((3,1))=1$. We depict the configuration $f$ with the below diagram. 


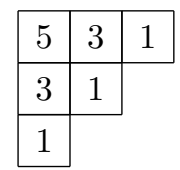

There is exactly one cell in the Young diagram $\lambda$, the cell $(1,1)$, that is contained in exactly one member of $\mathcal{F}=\left\{H_{r}: r \in \lambda\right\}$. Hence, $S=\{(1,1)\}$ and $\min (n, n-|S|+1)=$ $n-|S|+1$. With this in mind, set $n=6$ and, as $n-|S|+1=6-1+1=6$, assume that $m$ is an integer satisfying $1 \leq m \leq 5$. Suppose that there exists a surjective tableau $T$ of shape $\lambda$, and with entries from $[m]$, such that $T$ satisfies the configuration $f$ defined above. The cells $(1,1),(1,2)$ and $(2,1)$ are cells $r \in \lambda$ that satisfy $f(r)=h_{r}$. Moreover, because $T$ satisfies $f, f(r)=h_{r}$ implies that no two entries of $T$ in $H_{r}$ are the same and the entry of $T$ in cell $r$ is the $h_{r}^{t h}$ smallest element of the set of entries of $T$ that are contained in $H_{r}$.

So consider the cell $(2,2)$ of $\lambda$. Since $m \leq 5$, some two entries of $T$ in $H_{(1,1)}$ are the same, or the entry of $T$ in cell $(2,2)$ equals to the entry of $T$ in some other cell, $\left(i_{1}, j_{1}\right)$, in $\lambda$. Since $f((1,1))=5=h_{(1,1)}$, no two entries of $T$ in $H_{(1,1)}$ are the same. So the entry of $T$ in cell $(2,2)$ equals to the entry of $T$ in some other cell, $\left(i_{1}, j_{1}\right)$, in $\lambda$. If $\left(i_{1}, j_{1}\right)=(1,1)$, then the entry of $T$ in cell $(2,2)$ of $\lambda$ is larger than the entries of $T$ in cells $(1,2)$ and $(2,1)$ of $\lambda$. But that is impossible by the above. If $\left(i_{1}, j_{1}\right)=(2,1)$ or if $\left(i_{1}, j_{1}\right)=(3,1)$, then the entry of $T$ in cell $(2,1)$ of $\lambda$ is the $k^{t h}$ smallest element of the set of entries of $T$ that are contained in $H_{(2,1)}$ for some $k \leq 2$. But that is impossible by the above. By symmetry, it is impossible for $\left(i_{1}, j_{1}\right)=(1,2)$ or for $\left(i_{1}, j_{1}\right)=(1,3)$. Hence, we have reached a contradiction. It follows that there is no such surjective tableau $T$.

Example 3.24. Consider a skew shape $\lambda / \mu$ with $n$ cells, and let $S$ denote the set of cells of $\lambda / \mu$ that are contained in exactly one member of $\left\{H_{r}: r \in \lambda / \mu\right\}$. The elements of $S$ are also known as the outer corners of $\mu$. Clearly, there exists a standard skew tableau of shape $\lambda / \mu$. Corollary 3.11 implies that such a tableau exists if and only if for all integers $n-|S|+1 \leq m \leq n$ and for all configurations $f$ of $\lambda / \mu$, there exists a surjective tableau $T$ of shape $\lambda / \mu$, with $[m]$ as the set of entries of $T$, such that $T$ satisfies $f$.

\subsection{The average number of generalized tableaux}

Let $S(n, m)$ denote the Stirling number of the second kind, namely the number of set partitions of $[n]$ into $m$ parts. Let $\mathcal{F}$ be a family of subsets of $[n]$ that satisfies the marriage condition, let $m \in[n]$, and let $t$ be a transversal of $\mathcal{F}$. If $f$ is a configuration of $t$, then let $A_{n, m}(f)$ denote the number of surjective maps $\sigma:[n] \rightarrow[m]$ that satisfy $f$. Moreover, let $X$ be the set of configurations $f$ of $t$ such that $A_{n, m}(f) \geq 1$. Then define the average value of $A_{n, m}(f)$ over all configurations $f$ of $t$ satisfying $A_{n, m}(f) \geq 1$ to be

$$
\frac{1}{|X|} \sum_{f \in X} A_{n, m}(f)
$$

if $|X|>0$, and 0 otherwise.

Theorem 3.25. Let $\mathcal{F}$ be a flagged family of subsets of $[n]$ such that $|\mathcal{F}|=n$ and let $t$ be the transversal of $\mathcal{F}$. Moreover, let $S \subseteq[n]$ be the set of elements $k \in[n]$ such that $k \in F$ for exactly one member $F$ of $\mathcal{F}$, and let $m$ be an integer satisfying

$$
n-|S|+1 \leq m \leq n .
$$


Then the average value of $A_{n, m}(f)$ over all configurations $f$ of t satisfying

$$
A_{n, m}(f) \geq 1
$$

is

$$
\frac{m ! S(n, m)}{\prod_{F \in \mathcal{F}}|F|}
$$

Remark 3.26. Consider the sequence $\left(p_{k}(x)\right)_{k=0,1,2, \ldots}$ of polynomials in $\mathbb{Q}[x]$ such that $p_{0}(x)=1$ and, for all $k$,

$$
p_{k+1}(x)-p_{k+1}(x-1)=x p_{k}(x) .
$$

If $k=n-m$, then $S(n, m)=p_{k}(m)$ (see [2, 22]). So if $k$ is fixed, then we can compute closed-form expressions for $S(n, m)$. For instance, Expression 3.9 becomes

$$
\frac{m !}{\prod_{F \in \mathcal{F}}|F|}
$$

if $n=m$,

$$
\left(\begin{array}{c}
m+1 \\
2
\end{array}\right) \frac{m !}{\prod_{F \in \mathcal{F}}|F|}
$$

if $n=m+1$, and

$$
\frac{1}{2}\left(\begin{array}{c}
m+1 \\
2
\end{array}\right)\left(\left(\begin{array}{c}
m+1 \\
2
\end{array}\right)+\frac{2 m+1}{3}\right) \frac{m !}{\prod_{F \in \mathcal{F}}|F|}
$$

if $n=m+2$.

In order to prove Theorem 3.25, we prove the following.

Lemma 3.27. Let $m, n \in \mathbb{N}$ such that $m \leq n$, and let $\mathcal{F}$ be a family of subsets of $[n]$ that has a transversal $t: \mathcal{F} \rightarrow[n]$ such that $t$ is surjective. Then every surjective function $\sigma:[n] \rightarrow[m]$ satisfies exactly one configuration $f$ of $t$.

Proof. Let $\sigma:[n] \rightarrow[m]$ be a surjective map. Then $\sigma$ satisfies the configuration $f$ of $t$ defined by letting, for all $F \in \mathcal{F}, f(t(F))=k$ where $\sigma(t(F))$ is the $k^{t h}$ smallest element of the set $\sigma(F)$. Now, suppose that $\sigma$ satisfies more than one configuration of $t$. Then, let $f_{1}$ and $f_{2}$ be two distinct configurations of $t$. Because $f_{1} \neq f_{2}$ and because $t$ is surjective, there is an element $F \in \mathcal{F}$ such that $f_{1}(t(F)) \neq f_{2}(t(F))$. So write $k_{1}=f_{1}(t(F))$ and write $k_{2}=f_{2}(t(F))$. Since $\sigma$ satisfies $f_{1}$, Definition 3.4 implies that $\sigma(t(F))$ is the $k_{1}^{t h}$ smallest element of $\sigma(F)$. Moreover, since $\sigma$ satisfies $f_{2}$, Definition 3.4 implies that $\sigma(t(F))$ is the $k_{2}^{t h}$ smallest element of $\sigma(F)$. However, this is impossible because $k_{1}=f_{1}(t(F)) \neq f_{2}(t(F))=k_{2}$.

Now, we prove Theorem 3.25.

Proof. By Definition 3.4, the total number of configurations of $\mathcal{F}$ equals to $\prod_{F \in \mathcal{F}}|F|$. Moreover, it is well-known that the number of surjective maps from $[n]$ to $[m]$ is given by $m ! S(n, m)$. By Lemma 3.27, every surjective map satisfies exactly one configuration. Moreover, by Theorem 3.10, every configuration of $\mathcal{F}$ is satisfied by some surjective map from $[n]$ to $[m]$. From this, the theorem follows. 
Theorem 3.25 implies the following consequence relating to how the values $A_{n, m}(f)$ are distributed. By Theorem 3.10, every configuration $f$ of $t$ is satisfied by at least one surjective map $\sigma:[n] \rightarrow[m]$. Hence, by Theorem 3.25 and the fact that $A_{n, m}(f) \geq 1$ always holds, it follows that for all constants $k \geq 1$ the number of configurations $f$ of $t$ that satisfy

$$
A_{n, m}(f) \leq k \cdot \frac{m ! S(n, m)}{\prod_{F \in \mathcal{F}}|F|}
$$

is at least

$$
\left(1-\frac{1}{k}\right) \prod_{F \in \mathcal{F}}|F| .
$$

We now illustrate Theorem 3.25 with some examples and in the process describe its relationship with the hook-length formula.

Example 3.28. Let $\lambda=(6,5,4,3,2,1)$ and $\mu=(1)$. The skew shape $\lambda / \mu$ is depicted below.

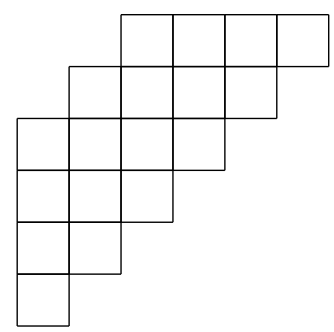

Since $\lambda / \mu$ has eighteen cells, let $n=18$. The cells of $\lambda / \mu$ that are contained in exactly one member of the family $\mathcal{F}_{\lambda / \mu}$ are $(1,3),(2,2)$, and $(3,1)$. Hence, $S=\{(1,3),(2,2),(3,1)\}$ and $n-|S|+1=n-2$. So let $m=n-2=16$. Then by Theorem 3.25 and Remark 3.26, the average value of $A_{n, m}(f)$ over all configurations $f$ of $\lambda / \mu$ satisfying $A_{n, m}(f) \geq 1$ is given by

$$
\begin{aligned}
\frac{1}{2}\left(\begin{array}{c}
m+1 \\
2
\end{array}\right)\left(\left(\begin{array}{c}
m+1 \\
2
\end{array}\right)\right. & \left.+\frac{2 m+1}{3}\right) \frac{m !}{\prod_{F \in \mathcal{F}_{\lambda / \mu}|F|}}= \\
& =\frac{1}{2}\left(\begin{array}{c}
16+1 \\
2
\end{array}\right)\left(\left(\begin{array}{c}
16+1 \\
2
\end{array}\right)+\frac{2 \cdot 16+1}{3}\right) \frac{16 !}{\prod_{r \in \lambda / \mu} h_{r}} \\
& =\frac{1}{2}\left(\begin{array}{c}
17 \\
2
\end{array}\right)\left(\left(\begin{array}{c}
17 \\
2
\end{array}\right)+11\right) \frac{16 !}{(7 \cdot 5 \cdot 3 \cdot 1)^{3} \cdot 5 \cdot 3 \cdot 1 \cdot 3 \cdot 1 \cdot 1} \\
& =4014814003+\frac{1}{5} .
\end{aligned}
$$

The hook-length formula, first proved by Frame, Robinson, and Thrall [8], is wellknown. It is as follows. A skew shape $\lambda / \mu$ is a straight shape if $\mu=\emptyset$. Given a Young diagram $\lambda$, call a standard skew tableau of straight shape $\lambda$ a standard Young tableaux of shape $\lambda$. If $\lambda$ is a Young diagram with $n$ cells, then the number of standard Young tableaux of shape $\lambda$ equals

$$
\frac{n !}{\prod_{r \in \lambda} h_{r}} \text {. }
$$


Moreover, the above formula was also proved by Edelman and Greene to equal the number of balanced tableaux of shape $\lambda$ [5]. Furthermore, the hook-length formula does not hold for skew shapes. Taking $m=n$ in Theorem 3.25, setting $\mathcal{F}=\mathcal{F}_{\lambda}$, and letting $t$ be the unique transversal of $\mathcal{F}$, we see that the average value of $A_{n, m}(f)$ over all configurations $f$ of $t$ satisfying $A_{n, m}(f) \geq 1$ equals to the number of standard Young tableau of shape $\lambda$.

Example 3.29. Let $\lambda=(6,5,4,3,2,1)$. The Young diagram $\lambda$ is depicted below.

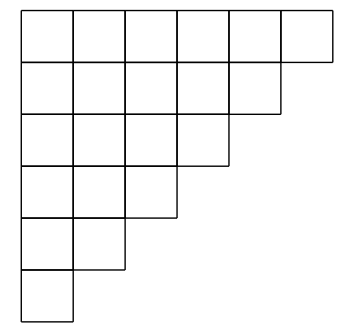

Since $\lambda$ has twenty-one cells, let $n=21$. The cell of $\lambda$ that is contained in exactly one member of the family $\mathcal{F}_{\lambda}$ is $(1,1)$. Hence, $S=\{(1,1)\}$ and $n-|S|+1=n$. So let $m=n=21$. Then by Theorem 3.25 and Remark 3.26, the average value of $A_{n, m}(f)$ over all configurations $f$ of $\lambda$ satisfying $A_{n, m}(f) \geq 1$ is given by the hook-length formula

$$
\begin{aligned}
\frac{m !}{\prod_{F \in \mathcal{F}_{\lambda}}|F|} & =\frac{21 !}{\prod_{r \in \lambda} h_{r}} \\
& =\frac{21 !}{1^{6} \cdot 3^{5} \cdot 5^{4} \cdot 7^{3} \cdot 9^{2} \cdot 11} \\
& =1100742656
\end{aligned}
$$

and is, by the hook-length formula, equal to the number of standard Young tableaux of shape $\lambda$.

Remark 3.30. Theorem 3.25 is versatile. For instance, possible applications of the special case of Theorem 3.25 in the case of permutations are as follows. There is a formula for the number of standard skew tableaux of shape $\lambda / \mu$, known as Naruse's formula. Asymptotic properties of Naruse's formula were analysed by Morales, Pak, and Panova in [17]. In particular, it turns out that in general, the number of standard skew tableaux of shape $\lambda / \mu$ divided by

$$
\frac{n !}{\prod_{r \in \lambda / \mu} h_{r}},
$$

where $n$ is the number of cells of $\lambda / \mu$, can be arbitrarily large. Hence, we can apply Theorem 3.25 to Naruse's formula and, using the work of Morales, Pak, and Panova in [17], analyse lower bounds on the number of configurations $f$ of $\lambda / \mu$ such that $A_{n, n}(f) \geq 1$ and $A_{n, n}(f)$ is strictly less than

$$
\frac{n !}{\prod_{r \in \lambda / \mu} h_{r}} .
$$

Remark 3.31. Regarding Remark 3.30, there are variants and generalizations of Naruse's formula, the formula mentioned in Remark 3.30, for skew shifted shapes [9, 19]. What we observe about these shapes is that the "hook-sets" for skew shifted shapes as defined 
in $[9,19]$ also form examples of flagged families. Hence, the results in this section can be replicated verbatim to include skew shifted shapes. Moreover, it is claimed by Morales, Pak, and Panova in [17] that their analysis of Naruse's formula can be extended to skew shifted shapes. It also appears that we can even extend the above to involve posets known as $d$-complete posets [19], as there is a generalization of Naruse's formula for such posets and the "hook-sets" in these formulas are a generalization of the "hook-sets" for the skew shifted shapes [19].

We conclude this subsection by asking some natural enumerative questions related to the quantity $A_{n, m}(f)$ in Theorem 3.25.

1. Which configurations $f$ as specified in Theorem 3.25 are such that $A_{n, m}(f)$ is given by Equation (3.9)?

2. Which flagged families $\mathcal{F}$ with transversal $t$ are such that $A_{n, m}(f)$, with $m$ fixed, is independent of the configuration $f$ of $t$ ?

3. If the configuration $f$ as specified in Theorem 3.25 is such that $f(F)=1$ for all $F \in \mathcal{F}$, when is $A_{n, m}(f)$ maximal, and can $A_{n, m}(f)$ be less than or equal to Equation (3.9)?

4. Let $\mathcal{F}$ be a flagged family and let $t$ be a transversal of $\mathcal{F}$. For $m$ fixed, which configurations $f$ of $t$ maximize or minimize the value of $A_{n, m}(f)$ ?

5. Does the value of $m$ in comparison to $n$ affect answers to any of the above questions?

\section{ORCID iDs}

\section{Brian Tianyao Chan (D) https://orcid.org/0000-0003-0525-1362}

\section{References}

[1] O. Angel, A. E. Holroyd, D. Romik and B. Virág, Random sorting networks, Adv. Math. 215 (2007), 839-868, doi:10.1016/j.aim.2007.05.019.

[2] M. Bóna, A walk through combinatorics, World Scientific Publishing Co., Inc., River Edge, NJ, 2002, doi:10.1142/4918, an introduction to enumeration and graph theory, With a foreword by Richard Stanley.

[3] G. J. Chang, On the number of SDR of a $(t, n)$-family, European J. Combin. 10 (1989), 231234, doi:10.1016/s0195-6698(89)80056-3.

[4] P. Edelman and C. Greene, Combinatorial correspondences for Young tableaux, balanced tableaux, and maximal chains in the weak Bruhat order of $S_{n}$, in: Combinatorics and algebra (Boulder, Colo., 1983), Amer. Math. Soc., Providence, RI, volume 34 of Contemp. Math., pp. 155-162, 1984, doi:10.1090/conm/034/777699.

[5] P. Edelman and C. Greene, Balanced tableaux, Adv. in Math. 63 (1987), 42-99, doi:10.1016/ 0001-8708(87)90063-6.

[6] N. J. Y. Fan, Standard Rothe tableaux, Discrete Math. 342 (2019), 3182-3193, doi:10.1016/j. disc.2019.06.027.

[7] S. Fomin, C. Greene, V. Reiner and M. Shimozono, Balanced labellings and Schubert polynomials, European J. Combin. 18 (1997), 373-389, doi:10.1006/eujc.1996.0109.

[8] J. S. Frame, G. d. B. Robinson and R. M. Thrall, The hook graphs of the symmetric groups, Canad. J. Math. 6 (1954), 316-324, doi:10.4153/cjm-1954-030-1. 
[9] W. Graham and V. Kreiman, Excited Young diagrams, equivariant $K$-theory, and Schubert varieties, Trans. Amer. Math. Soc. 367 (2015), 6597-6645, doi:10.1090/ s0002-9947-2015-06288-6.

[10] M. Hall, Jr., Distinct representatives of subsets, Bull. Amer. Math. Soc. 54 (1948), 922-926, doi:10.1090/s0002-9904-1948-09098-x.

[11] P. Hall, On representatives of subsets, J. London Math. Soc 10 (1935), 26-30, doi:10.1112/ jlms/s1-10.37.26.

[12] J. L. Hirst and N. A. Hughes, Reverse mathematics and marriage problems with unique solutions, Arch. Math. Logic 54 (2015), 49-57, doi:10.1007/s00153-014-0401-z.

[13] J. L. Hirst and N. A. Hughes, Reverse mathematics and marriage problems with finitely many solutions, Arch. Math. Logic 55 (2016), 1015-1024, doi:10.1007/s00153-016-0509-4.

[14] L. Hurwicz and S. Reiter, Transversals, systems of distinct representatives, mechanism design, and matching, in: Markets, Games, and Organizations, Springer, Berlin, Heidelberg, 2003, doi:10.1007/978-3-540-24784-5_10.

[15] M. Konvalinka, A bijective proof of the hook-length formula for skew shapes, European J. Combin. 88 (2020), 103104, 14, doi:10.1016/j.ejc.2020.103104.

[16] D. P. Little, Combinatorial aspects of the Lascoux-Schützenberger tree, Adv. Math. 174 (2003), 236-253, doi:10.1016/s0001-8708(02)00038-5.

[17] A. H. Morales, I. Pak and G. Panova, Asymptotics of the number of standard Young tableaux of skew shape, European J. Combin. 70 (2018), 26-49, doi:10.1016/j.ejc.2017.11.007.

[18] A. H. Morales, I. Pak and G. Panova, Hook formulas for skew shapes I. $q$-analogues and bijections, J. Combin. Theory Ser. A 154 (2018), 350-405, doi:10.1016/j.jcta.2017.09.002.

[19] H. Naruse and S. Okada, Skew hook formula for $d$-complete posets via equivariant $K$-theory, Algebr. Comb. 2 (2019), 541-571, doi:10.5802/alco.54.

[20] P. F. Reichmeider, The equivalence of some combinatorial matching theorems, Polygonal Publ. House, Washington, NJ, 1984, doi:10.2307/3615712.

[21] R. P. Stanley, Enumerative combinatorics. Vol. 2, volume 62 of Cambridge Studies in Advanced Mathematics, Cambridge University Press, Cambridge, 1999, doi:10.1017/ cbo9780511609589, with a foreword by Gian-Carlo Rota and appendix 1 by Sergey Fomin.

[22] R. P. Stanley, Enumerative Combinatorics. Volume 1, volume 49 of Cambridge Studies in Advanced Mathematics, Cambridge University Press, Cambridge, 2nd edition, 2012, doi: 10.1017/cbo9781139058520.

[23] F. Viard, A new family of posets generalizing the weak order on some coxeter groups, 2015, arXiv:1508.06141 [math.CO].

[24] F. Viard, A natural generalization of balanced tableaux, 2016, arXiv:1407.6217v2 [math.CO]. 